\title{
Schreiben gegen die Unsichtbarkeit
}

\section{Der Roman En famille von Marie NDiaye}

Cornelia RuHe

Esse est percipi.

GEORGE BERKELEY ${ }^{1}$

Frankreich verfügt über eine lange republikanische Tradition. Das Prinzip der égalité, der Gleichheit gehört zu den Grundpfeilern dieser Republik. Unter Berufung auf die Charta der Menschenrechte erließ Frankreich darüber hinaus im Jahr 1972 die „loi Pléven“, ein Gesetz gegen den Rassismus. Gleichbehandlung und Schutz vor Diskriminierung sollten somit eine Selbstverständlichkeit sein. In der Realität bleibt dies nicht nur meist ein Ideal, sondern gerade aus diesem Gesetz wurden durchaus auch problematische Konsequenzen gezogen.

Der Forderung nach Gleichbehandlung ist es beispielsweise zu verdanken, dass, so der Sozialwissenschaftler und Politologe Pap Ndiaye, im gesamten 20. Jahrhundert keine separaten statistischen Werte über die schwarze Bevölkerung Frankreichs erhoben wurden: „Le dernier recensement de la population noire de France date de... 1807.“ (Pap Ndiaye 2008: 76) Die Logik dieses Mangels ist ebenso offensichtlich wie fraglich: Was

1 George Berkeley: A Treatise Concerning the Principles of Human Knowledge. 1710. $§ 2$. 
gleich zu sein hatte - die gesamte französische Bevölkerung -, durfte folglich nicht durch die Statistik als unterschiedlich erfasst werden. ${ }^{2}$

Auch die französische Sozialwissenschaft scheute viele Jahre davor zurück, sich mit der Situation der Schwarzen in Frankreich auseinanderzusetzen. Für diese Zurückhaltung gibt es, so erläutert Pap Ndiaye, abgesehen vom Fehlen statistischer Daten, mehrere Gründe: Neben der Angst vor einer Essentialisierung im Sinne des Begriffs der Hautfarbe, dem der Ruch des Rassismus anhängt, würde eine solche Untersuchung mit dem klassenkämpferischen Impetus brechen, der in der französischen Soziologie lange die Wissenschaftslandschaft bestimmt hat (Pap Ndiaye 2008: 77f.).

Ndiaye zeigt in seinem Text, dass es in Frankreich eine Tradition des abwertenden Umgangs mit der schwarzen Bevölkerung gibt, die sich mit den neuen Gesetzen zur Antidiskriminierung in den 1970er Jahren nicht wesentlich geändert hat. Die Gesetze bekräftigten vielmehr, wie Ndiaye zeigt, ein eklatantes Missverhältnis zwischen dem zur Norm erhobenen Ideal des republikanischen Selbstverständnisses und der Realität der französischen Gesellschaft. Das Resultat ist ein Paradoxon: Obwohl die Bevölkerungsgruppen, die aus unterschiedlichen Phasen der Immigration hervorgegangen sind, im französischen Alltag omnipräsent sind, sind sie statistisch und juristisch nicht existent. Selbst für die Forschung, die häufig von öffentlichen Geldern abhängig ist, werden sie damit unzugänglich und somit ebenfalls nachgerade unsichtbar. ${ }^{3}$ Die Möglichkeit zur Partizipation an

2 Eine kurze Phase differentialistischer Rhetorik, die, wie Roger Brubaker richtig anmerkt, von der französischen Rechten vereinnahmt wurde, folgte eine „Rückkehr des Assimilationsgedankens“, die selbst in wissenschaftlichen Kontexten allenfalls einen gemäßigten Differentialismus zulässt (Brubaker: 2007: 173f.).

3 Die Problematik dieses Themas zeigt sich in geradezu exemplarischer Weise an der Diskussion des Buches Le déni de la culture von Hugues Lagrange (Paris 2010): Seine Thesen, die auf jahrelangen Forschungen basieren, stellen einen engen Zusammenhang zwischen ethnischer Herkunft und Delinquenz, insbesondere in der jugendlichen schwarzen Bevölkerung Frankreichs, dar. Von Wissenschaftlern wie Pap Ndiaye werden sie scharf kritisiert, insbesondere in Bezug auf eine dadurch drohende, tendenziell historisch unpräzise Essentialisierung des Kulturbegriffs (siehe hierzu „Débat Hugues Lagrange, Pap Ndiaye: délinquance et origines ethniques sont-elles liées?“ In: http://www.telerama.fr/ 
diesem Alltag, ihr Zugang zu ihrer Ausbildung angemessenen Arbeitsstellen, zu Wohnungen etc. ist allerdings nicht derselbe wie der anderer Gruppen mit einer historisch weiter zurückreichenden und damit längst normalisierten Migrationsgeschichte, die heute nicht mehr als solche wahrgenommen wird.

Im Folgenden soll es weniger um die konkrete Natur der Diskriminierungen gehen, denen die schwarze Bevölkerung Frankreichs ausgesetzt ist, als vielmehr um Literatur. Mechanismen der Ausschließung, wie sie hier angesprochen wurden, liegen als Thema auch den Romanen Marie NDiayes zugrunde. ${ }^{4}$ Es handelt sich bei ihren Texten um die äußerst subtile und konzise literarische Auslotung eines Misstandes, der nicht nur die französische Gesellschaft, sondern auch die Autorin selbst affiziert.

\section{VON VÄTERN UND WURZELN}

Marie NDiaye ist seit nunmehr über zwanzig Jahren eine von der Kritik geschätzte, vielfach preisgekrönte und in zahlreiche Sprachen übersetzte französische Autorin. Ihr erster Roman wurde im renommierten Pariser Verlag Les Éditions de Minuit veröffentlicht, als sie erst 17 Jahre alt war. Entsprechend blickte sie bereits auf eine 25jährige Karriere als Autorin zurück, als ihr im Jahr 2009, als vorläufige Krönung ihrer bisherigen Laufbahn mit dem Prix Goncourt der wichtigste französische Literaturpreis verliehen wurde. Bereits 2003 hatte die Comédie française ihr Theaterstück Papa doit manger in ihr Repertoire aufgenommen, eine Ehre, die vor ihr keiner Frau zu ihren Lebzeiten zuteil geworden ist.

Der Vater von Pap und Marie NDiaye ist Senegalese, hat die Familie aber verlassen, als beide Kinder noch sehr klein waren. Wie die Autorin selbst wiederholt betont hat, sieht sie sich als Teil der französischen Gesellschaft und der französischen Literatur; sie verfügt über keinerlei afrikani-

idees/delinquances-et-origines-ethniques-sont-elles-liees,61089.php (letzter Zugriff: 30.06 .11$)$ ).

4 Pap Ndiaye und Marie NDiaye sind Geschwister. Die beiden haben sich für unterschiedliche Schreibweisen ihres Nachnamens entschieden. Siehe hierzu Devarrieux, Claire (2009): „Enfant prodige, auteur prodigue“. In: Libération vom 03.11.2009. 
sche Wurzeln, da sie in einem ,rein ' französischen Kontext aufwuchs. Presse und Kritik versuchen ungeachtet dieser Aussagen immer wieder, NDiayes Verbundenheit mit afrikanischen Erzähltraditionen oder Themen nachzuweisen. Am Beispiel der Reaktionen auf ihre Publikationen und am Umgang mit ihr als öffentlicher Person wird exemplarisch deutlich, wie stark die Problematik von Integration und Assimilation Frankreich nach wie vor auf allen Ebenen prägt.

In Rezensionen der Texte Marie NDiayes, insbesondere aber auch in der Auseinandersetzung mit ihrer Person werden Interviewpartner und Journalisten nicht müde, Fragen nach ihren afrikanischen Wurzeln, typisch afrikanischen Motiven in ihren Texten und ihrer angeblich hybriden, franko-senegalesischen Identität zu stellen, obgleich die Autorin nach eigenen Angaben zu Afrika allenfalls oberflächliche Beziehungen im Rahmen allgemeiner Kenntnisse fremder Kulturen unterhält. ${ }^{5}$

Die Verleihung des Prix Goncourt an Marie NDiaye wurde in der internationalen Presse intensiv kommentiert. Der britische Guardian wies bereits im Titel auf die Hautfarbe der Autorin hin (,Black woman wins Prix Goncourt for the first time ${ }^{\text {“6 }}$ ), ihr Name folgte erst im Untertitel. Der Artikel, der zwar nicht unerwähnt ließ, dass NDiaye sich nicht auf afrikanische Wurzeln beruft, widmete dennoch diesem Aspekt einen beachtlichen Teil seines Textes. Auch andere anglophone Zeitungen bezeichneten sie hartnäckig als „French-Senegalese author“.

51991 wird NDiaye von einem Wissenschaftler gebeten, einen Fragebogen auszufüllen, der an eine Reihe frankophoner Autoren versandt worden ist. In ihrem Antwortbrief, der inzwischen im Internet veröffentlicht wurde, stellt sie klar, dass ,,[...] je ne puis être considérée comme une romancière francophone, c'està-dire une étrangère de langue française, aucune culture africaine ne m'a été transmise, je la connais, un peu, comme peuvent la connaître des personnes intéressées par toutes les formes de culture. Il me semblait important de le préciser, ne sachant si vous étudiez aussi des romancières aussi superficiellement africaines que je le suis.“ (NDiaye, Marie. „Une lettre“ (1992). In: http://aflit.arts. uwa.edu.au/NDiayeMarie.html (letzter Zugriff: 14.06.10)).

6 Flood, Alison (2009): „Black women wins Prix Goncourt for the first time“. In: The Guardian vom 02.11.2009.

7 So etwa Kellogg, Carolyn (2009): „Author Marie NDiaye is the first black woman to win the Prix Goncourt“. In: Los Angeles Times vom 02.11.2009. 
Während deutsche Zeitungen in dieser Hinsicht deutlich zurückhaltender sind, ${ }^{8}$ konstatierte der spanische El Pais überraschenderweise nicht nur, die Autorin sei „francesa de origen senegalésa“, sondern auch ihr Roman sei ,en cierta manera autobiográfica“. ${ }^{9}$ Die Presseagentur AFP hatte ebenfalls Interessantes über Marie NDiaye zu melden: „Like much of her work, the book touches on the troubled ties between Africa and its former colonial rulers". 10

Die senegalesische Tageszeitung Le soleil schließlich wusste es gar besser als die Autorin selbst:

„Même si elle ne le reconnaît pas toujours [...], la romancière Marie Ndiaye [sic! ...] est quelque peu écartelée entre deux cultures. [...] au fond d'elle, Marie Ndiaye a quand même quelque chose d'Africaine, de Sénégalaise. [...] Elle [...] fera sans doute l'effort de (re)venir régulièrement au Sénégal, pays d'origine de son père, pour mieux s'imprégner des réalités d'un continent où, peut-être sans le savoir, elle a tiré la force de l'âme qui a fait d'elle un écrivain si talentueux. “11

Ungeachtet der Tatsache, dass die Autorin selbst von Beginn ihrer Karriere an deutlich gemacht hat, dass sie keine emotionale oder kulturelle Bindung

8 Stattdessen wird in den Vordergrund gestellt, dass Marie NDiaye inzwischen in Deutschland lebt, so etwa „Marie NDiaye erhält Prix Goncourt“. In: Die Zeit vom 2.11.2009; „Prix Goncourt für Marie NDiaye“. In: Focus vom 02.11.2009; „Starke Frauen. Prix Goncourt für Marie NDiaye“. In: Frankfurter Allgemeine Zeitung vom 03.11.2009, S. 31. Die FAZ veröffentlicht wenige Wochen später ein ausführliches und sehr gelungenes Interview mit der Autorin: Bender, Niklas (2009): „Glauben Sie an Magie, Madame? Im Gespräch: Marie NDiaye“. In: Frankfurter Allgemeine Zeitung vom 05.12.2009, Z6.

9 Jiménez Barca, Antonio (2009): „La autora francosenegalesa Marie NDiaye se alza con el Goncourt“. In: El país vom 02.11.2009. Auch El mundo attestiert dem Roman autobiographische Züge (,La escritora Marie Ndiaye gana el premio Goncourt“. In: El mundo vom 02.11.2009).

10 „French-Senegalese woman scoops top French book prize“. In: afp.com, November 2009 (letzter Zugriff: 14.06.10).

11 Faye, Modou Mamoune (2009): „Ce Sénégal qui l'inspire“. In: Le soleil.sn, o.D. (letzter Zugriff: 14.06.10). 
an den Senegal im Besonderen oder Afrika im Allgemeinen hat, suchte die Kritik immer wieder nach entsprechenden Bezügen.

Es ist daher vermutlich nicht als Zufall zu werten, dass ausgerechnet Trois femmes puissantes, der erste Roman NDiayes, der sich tatsächlich mit Afrika beschäftigt, einen prestigereichen Literaturpreis davonträgt und darüber hinaus ein Publikumserfolg wird. Erst in dem Moment, in dem sie erfüllt, was die Presse stets von ihr erwartet zu haben scheint, wird ihr Erfolg zuteil. NDiaye selbst allerdings erläuterte in einem Interview, dass Afrika für sie nur ein Motiv des Textes sei: „,...] la présence de l'Afrique était pour moi juste un motif musical, qui lierait les trois parties. “12

Die Reaktionen in der französischen Presse variierten stark, je nach politischem Hintergrund der jeweiligen Zeitung: Le monde widmete ihr ausführliche Artikel, in denen die künstlerischen Qualitäten des Buches und die Kontinuität ihrer literarischen Karriere hervorgehoben wurden. ${ }^{13}$ Le Figaro hingegen schrieb ihren Namen gleich im Titel des Artikels falsch (Ndiaye statt NDiaye) und sprach von einem ,léger avantage“, den NDiaye aufgrund der Tatsache, dass sie eine Frau sei, vor ihren Konkurrenten gehabt habe.$^{14}$ Der Preis sei also nicht verdient, so wird unterstellt, sondern lediglich politischem Kalkül geschuldet. Auf diese und ähnliche Kommentare reagierte Libération, die sich über den Erfolg einer Autorin freute ,aimée par Libération depuis ses premiers pas littéraires“: „Que Marie NDiaye soit plutôt noire et plutôt femme n'ajoute rien, ni ne retranche, à ses qualités d'écrivain". ${ }^{15}$

Es ist bemerkenswert, wie stark die Artikel in der Presse sich nicht mit dem literarischen Werk, sondern mit der Person Marie NDiayes auseinandersetzen und dabei gerade ihrer Herkunft und kulturellen Zugehörigkeit besondere Aufmerksamkeit widmen. Die offensichtliche Unfähigkeit, die Texte ohne Ansehen ihrer Hautfarbe zu betrachten, prägt den öffentlichen

12 Crom, Nathalie (2009): „Marie NDiaye: ,Je ne veux plus que la magie soit une ficelle““. In: Télérama vom 13.11.2009.

13 Le monde vom 04.11.2009: Titelseite, „Marie NDiaye, Prix Goncourt, une force de l'écriture“ (mit Photo), S. 20, „Libre d'écrire“, S. 25, „Le jury du Goncourt récompense, dès le premier tour, Marie NDiaye“.

14 „Ndiaye remporte le Goncourt, Beigbeder le Renaudot“. In: Le figaro vom 03.11.2009.

15 „Puissante“. In: Libération vom 03.11.2009. 
Diskurs über die Autorin, deren Texte sich in sehr viel subtilerer Weise als es die Kritik wahrnimmt mit dem Thema der Ausschließung auseinander setzen. ${ }^{16}$

16 Damit nicht genug, entspann sich kurz nach der Preisverleihung eine heftige politische Kontroverse um ihre Person. Ein mehrere Monate altes Interview mit der Zeitschrift Les inrocks, in dem die Autorin sich über die Beweggründe für ihren Umzug nach Berlin geäußert hatte, wurde von der politischen Rechten wiederentdeckt. Auf die Frage, ob sie sich im Frankreich Sarkozys wohl fühle, antwortete NDiaye: ,Je trouve cette France-là monstrueuse. Le fait que nous [...] ayons choisi de vivre à Berlin depuis deux ans est loin d'être étranger à ça. Nous sommes partis juste après les élections, en grande partie à cause de Sarkozy, même si j'ai bien conscience que dire ça peut paraître snob. Je trouve détestable cette atmosphère de flicage, de vulgarité... Besson, Hortefeux, tous ces gens-là, je les trouve monstrueux.“ (Kaprielian, Nelly (2009): „L'écrivain Marie NDiaye aux prises avec le monde“. In: Les inrocks vom August 2009). Der konservative Abgeordnete Eric Raoult von der Regierungspartei UMP wandte sich aufgrund dieser Äußerung nach der Verleihung des Goncourt in einem offenen Brief an den Kultursminister, Frédéric Mitterand. Er forderte ihn auf, Marie NDiaye zur Ordnung zu rufen. Raoult appellierte dabei an den ,[...] devoir de réserve, dû aux lauréats du Prix Goncourt. En effet, ce prix qui est le prix littéraire français le plus prestigieux est regardé en France, mais aussi dans le monde, par de nombreux auteurs et amateurs de la littérature française. [...] A ce titre, le message délivré par les lauréats se doit de respecter la cohésion nationale et l'image de notre pays. Ces propos d'une rare violence, sont peu respectueux voire insultants, à l'égard de ministres de la République et plus encore du Chef de l'État. Il me semble que le droit d'expression, ne peut pas devenir un droit à l'insulte ou au règlement de compte personnel. Une personnalité qui défend les couleurs littéraires de la France se doit de faire preuve d'un certain respect à l'égard de nos institutions, plus de respecter le rôle et le symbole qu'elle représente.“ (Zitiert nach Leménager, Grégoire (2009): „Eric Raoult rappelle Marie NDiaye à son ,devoir de réserve““. In: Le Nouvel Observateur vom 09.11.2009). Die Presse verteidigte Marie NDiaye und das Recht auf Meinungsfreiheit - auch für Preisträger - vehement. Die öffentlichen Reaktionen waren auch international befremdet bis empört. Der Kultusminister konnte sich jedoch zu keiner entschiedenen Reaktion durchringen, sondern gab an, sich in seiner Rolle als Minister nicht für diese Auseinandersetzung zuständig zu fühlen 


\section{EN fAMILLE}

Der Titel des Romans von 1992 nutzt eine positiv affektiv konnotierte Wendung (,en famille: être avec les siens“"17), die bei der Lektüre des Textes schnell widerlegt wird. ${ }^{18}$ Zugleich evoziert er als Metonymie für das Gemeinwesen jedoch auch die zentrale Rolle, die der Familie in den Debatten um Assimilations- und Integrationspolitik zugewiesen wird. ${ }^{19}$

Die Erfahrung einer subtilen Form der Ablehnung und Abwertung in einem Land, das sich den Kampf gegen Diskriminierung und Rassismus seit langem offiziell auf die Fahnen geschrieben, mit der Umsetzung aber notorische Schwierigkeiten hat, prägt zahlreiche Texte NDiayes. Zwar spielt die Frage der Hautfarbe in ihren Romanen und Erzählungen auf vordergründiger Ebene keine Rolle, ihre Protagonisten weisen jedoch häufig nicht näher definierte ,Besonderheiten“ auf, die sie von ihren Mitmenschen unterscheiden. In ihren Texten geht es daher häufig um die Auseinandersetzung mit der Andersartigkeit der Protagonist(inn)en, die von der Gesellschaft aus schwer fassbaren und in den Texten nicht konkret benannten Gründen abgelehnt und ausgestossen werden.

Der Roman En famille, der im Folgenden untersucht werden soll, kann in dieser Hinsicht als exemplarisch gelten.

$\mathrm{Zu}$ Beginn des Textes kehrt die zunächst namenlose Protagonistin in das Dorf und in das Haus ihrer Familie zurück, wo gerade eine Familienfeier stattfindet. Die Familie gibt zunächst vor, sie nicht zu kennen. Ihre Tante

(„Frédéric Mitterrand ne souhaite pas ,arbitrer“ la polémique NDiaye-Raoult“. In: Le monde vom 12.11.2009). Es ist bezeichnend, dass diese Polemik nicht in dem Moment aufkam, als die Aussage gemacht wurde, sondern erst, als die Autorin aufgrund des erhaltenen Preises ins Rampenlicht rückte.

17 Le nouveau petit Robert. Dictionnaire de la langue française. Paris 2000, S. 998a.

18 Der Titel des Romans evoziert für den französischen Leser das eponyme Kinderbuch von Hector Malot (1893). Zu diesem und anderen intertextuellen Bezügen bereitet die Verfasserin eine eigene Publikation vor.

19 Vgl. hierzu auch die Bemerkungen von Laura Ann Stoler zur Rolle der Familie in den Métissage-Diskursen in den französischen Kolonien: „Children had to be taught both their place and race, and the family was the crucial site in which future subjects and loyal citizens were to be made.“ (Stoler 1997: 203) 
Colette gibt ihr schließlich den Namen Fanny und lässt sie nur widerwillig ein. Fanny ist gekommen, um der Familie ihren Entschluss mitzuteilen, nach der seit Jahren verschwundenen Tante Leda zu suchen, von deren Rückführung in den Schoß der Familie sie sich auch ihre eigene Versöhnung mit ihr erhofft. Die Familie lehnt Fanny aus nicht näher genannten Gründen ab, die mit ihrer Person zu tun zu haben scheinen, die sie ihrerseits in einem Fehlverhalten ihrer Eltern oder ihrer selbst sucht. Gemeinsam mit ihrem widerstrebenden Cousin Eugène, den sie sich als Reisegefährten auserkoren hat, bricht sie auf zu einer Odyssee durch die angrenzenden Dörfer. Auch das Dorf ihres Vaters sucht sie auf, das zwar nur eine kurze Busfahrt entfernt liegt, in dem aber ein anderes Klima herrscht und auch Architektur und Kleidung der Menschen sich stark von den anderen Dörfern unterscheiden. Eugène gibt die Suche bald auf, Fanny zieht alleine weiter, verdingt sich eine Zeitlang als Küchenhilfe, dann als Kellnerin in einem Lokal. Ihre Suche nach Leda bleibt erfolglos. Sie verbringt kurze Zeit bei ihrer Mutter, die an der Peripherie einer großen Stadt lebt. Dort ist Fanny anerkannt, die anderen Bewohner ähneln ihr. Dennoch verlässt sie diesen Ort wieder, um in das Dorf ihrer Familie zurückzukehren und eine Zeitlang unbemerkt in einem Winkel im Anwesen ihrer Großmutter zu hausen. Bei der Hochzeit ihres Cousins Eugène wird sie jedoch entdeckt und von dessen Hund zerfleischt. Ihre Überreste werden achtlos auf den Misthaufen geworfen, das Fest geht weiter. Wenig später findet Tante Colette bei einem Waldspaziergang Fanny wieder, sie ist nackt und ganz offensichtlich eine verbesserte Version ihres alten Selbst, da sie sich nun in nichts mehr von den anderen Mitgliedern der Familie unterscheidet. Sie findet vorübergehend Einlass in das Anwesen der Familie. Ihre Bemühungen, sich im Dorf einbürgern zu lassen, schlagen fehl, ihre Mutter verstößt sie aufgrund der Wandlung, die sie durchgemacht hat. Fanny lässt sich schließlich am Rande des Dorfes nieder, wo sie recht erfolgreich dem Beruf der Prostituierten nachgeht. Den Aufforderungen ihrer Familie, nun doch noch ihren Cousin Eugène zu heiraten, kommt sie zunächst nicht nach. Tante Colette findet sie schließlich als nahezu durchsichtige und offensichtlich kurz vor der völligen Auflösung stehende Erscheinung auf ihrem Treppenabsatz. Wenig später fährt Tante Colette mit ihrem Sohn Eugène zu Fannys Vater, um ihn darum zu bitten, den untüchtigen Sohn unter seine Obhut zu nehmen und ihm zu helfen, seinen Platz im Leben zu finden. 


\section{FAMILIEN/NAMEN}

Über eine äußerliche Besonderheit der Protagonistin erfährt man im Text nichts, es wird aber sehr deutlich, dass sie sich von den übrigen Familienangehörigen in gewisser Weise unterscheiden muss. Im Text heißt es, sie verfüge über ,[de] nombreuses particularités inhérentes à la silhouette, au langage, à la forme de l'esprit de Fanny“ (S. 87). Diese Andersartigkeit scheint es auch zu sein, die sie zur ,étrangère“ (S. 156) in der Familie macht. Der Roman lässt bewusst offen, was die Eigenschaft ist, die die Protagonistin von den Anderen trennt. Ist es ihre „silhouette“, ihre Sprache, sind es ihre Verhaltensweisen oder gar lange zurückliegende Verfehlungen in der Familiengeschichte, die sich auswirken? Zwar ist es naheliegend davon auszugehen, dass sie über eine dunkle Hautfarbe verfügt, wichtig ist letztlich jedoch nur, dass sie ausgegrenzt wird, weil sie sich von den Anderen unterscheidet. Die Ausgrenzung liegt im Blick des Betrachters begründet, der seine Kriterien jedoch nie preisgibt bzw. von Fall zu Fall ändert.

Die Leerstelle, die Marie NDiayes raffinierte Textstrategie damit etabliert, lässt erkennen, dass zwischen den von ihr beschriebenen Exklusionsmechanismen und dem Rassismusbegriff, wie er von der neueren anthropologischen Forschung definiert wird, enge Verbindungen bestehen:

„Racism is commonly understood as a visual ideology in which somatic features are thought to provide the crucial criteria of membership. But racism is not really a visual ideology at all; physiological attributes only signal the nonvisual and more salient distinctions of exclusion on which racism rests. “20

Ebensowenig wie die Besonderheit der Protagonistin im Text definiert wird, wird auch ihr tatsächlicher Name genannt. Es wird erwähnt, ,que personne, dans la famille, n'a jamais su prononcer [son prénom] correctement, mis à part l'aïeule“ (S. 77), er sei ,d'ailleurs si curieux que vous éprouveriez les plus grandes difficultés à l'entendre, puis à le prononcer“" (S. 91). Der Name ist folglich so fremd für den dörflichen französischen Kontext, in dem der Roman spielt, dass man ihn dort nicht nur kaum aussprechen kann, sondern schon sein Klang Schwierigkeiten bereitet. Fanny sieht es als Fehler ihrer ,parents étourdis“ (S. 139) an, ihr diesen offensichtlich exotischen

20 Stoler: 1997: 203. 
Namen gegeben zu haben, der ihre Fremdheit nur noch stärker betont. Stattdessen, so suggeriert der Text, hätte man sie mit einem Namen versehen sollen, der ihre Fremdheit kaschiert, der sie unauffällig macht.

Ihre Verwandten erkennen ihren Namen nicht einmal als den eines Menschen an:

„Ces syllabes sonnent incongrûment à nos oreilles, aussi aurions-nous tendance à trouver bien laid et compliqué, ce prénom qui, pour nous, ne peut en être véritablement un. Il se rapproche davantage du nom ou du sobriquet que nous accorderions à un chien ou à un chat. Il n'est pas nécessaire que le nom des bêtes ait un sens ni qu'il rappelle quelque chose, et jamais il ne doit évoquer quelque personne de la famille: le vrai prénom de Fanny ne peut nous faire penser à rien.“ (S. 295)

Damit, dass ihr richtiger Name mit dem eines Haustiers verglichen wird, wird offensichtlich, was die Position der Protagonistin in der Familie ist. Nicht nur der Status der Familienangehörigen, sondern selbst der des Menschen wird ihr dadurch implizit abgesprochen.

Ihr Name weist die Protagonistin folglich von Anfang an als fremd aus, so dass sie ihn nur allzu bereitwillig aufgibt: Sie habe, so sagt sie, ohnehin einen neuen Namen gebraucht (S. 9). Dieser neue Name, Fanny, trägt zwar nicht mehr die kulturelle Fremdheit, die der ,alte " Name offensichtlich transportierte, ist aber auch kein gängiger französischer Vorname. Er ist vielmehr, wie die Erzählerinstanz der Anfangspassage kommentiert, ein Name, der nur ,in Büchern anzutreffen“ ist (S. 10). Wenn damit auch die Fremdheit ihres ursprünglichen Namens getilgt ist, so bedeutet der neue Name zugleich eine andere, grundlegendere Form der Ausgrenzung: Fanny wird dem Bereich des Fiktiven zugewiesen. Denn Fanny ist zum Beispiel der Titel von zwei bekannten französischen Texten, dem Skandalroman von Ernest Feydeau von 1858 und dem Theaterstück von Marcel Pagnol von 1931. Die Entwirklichung, die sich in dieser Umbenennung andeutet, wird später im Roman konkrete Formen annehmen.

Nur wenige kennen Fannys eigentlichen Namen und sprechen sie so an. Dazu gehören die ,aïeule“, die Großmutter Fannys und Matriarchin der Familie, aber auch Fannys Verlobter George. An seine Existenz erinnert Fanny sich allerdings erst, als er sie auf dem Dorf aufsucht. Es ist ihr unangenehm, dass er sie mit ihrem richtigen Namen anspricht: 
„Et il l'appela de son ancien prénom en suçant longuement, avec tendresse, chaque consonne. „Je ne veux plus entendre cela!' s'exclama Fanny effrayée. Elle regarda prudemment autour d'elle; dans le brouhaha et l'agitation du déjeuner, les paroles de Georges n'avaient pas dû atteindre d'autres oreilles que les siennes.“ (S. 116)

Die Erwähnung ihres eigentlichen Namens könnte, so fürchtet Fanny, ihre mühsam erkämpfte Position in dem Gasthaus, in dem sie arbeitet, gefährden. Für sie ist der neue Name eine Möglichkeit, ihre Fremdheit zu kaschieren, auch wenn diese keineswegs nur daran abzulesen ist, wie aus den Reaktionen der Mitmenschen offensichtlich wird.

\section{Ins Herz des LANDES}

Fanny kehrt mit George an die Peripherie der Großstadt zurück, wo er und auch Fannys Mutter leben und wo Fanny selbst aufgewachsen ist. Dort wird sie überaus freundlich von seiner Familie aufgenommen. Seine Mutter und seine Schwestern bemühen sich sehr um sie, selbstverständlich nennen sie sie bei ihrem richtigen Namen, der wiederum nicht zitiert wird. Während ihres Aufenthaltes bei ihnen verdingt Fanny sich in einem Fast-FoodRestaurant. Ebenso wie Georges und seine Familie ähneln auch die anderen Angestellten dort Fanny, ,lui ressemblant par de nombreux traits, au point qu'une illusion lui faisait voir souvent, en un collègue quelconque, Georges ou elle-même“ (S. 139). Einige der weiblichen Angestellten tragen ebenfalls den Namen, den die Protagonistin so sorgfältig verheimlicht. Sie befindet sich folglich hier, am Rande der Stadt, in einem Kontext, in dem sie nicht auffällt, vielmehr wird sie geschätzt, weil sie die hier von ihr erwarteten Leistungen in hervorragender Weise erbringt. Sie wird akzeptiert, weil sie Funktionen erfüllt, die ihr und ihresgleichen zugestanden werden und die der Konsumgesellschaft nützen. Die Peripherie der Großstadt wird ihr unter diesen Bedingungen als ihr natürlicher Ort zugewiesen, hier muss sie nicht um ihre Akzeptanz oder ihre soziale Position bangen. Die Gesellschaft, die das Buch schildert, ist eine sorgfältig segregierte, in der äußerliche Merkmale für die Akzeptanz auf bestimmten Ebenen entscheidend sind.

Für Fanny ist jedoch klar, dass ihr damit nicht die erstrebte gesellschaftliche Zugehörigkeit gewährt wird. Entsprechend empfindet sie es als gera- 
dezu beleidigend, mit den Bewohnern der Peripherie verglichen zu werden, hat sie doch den Eindruck, sich längst über sie erhoben zu haben. ${ }^{21}$ Ihre Rebellion richtet sich damit gegen die Segregation, gegen eine Essentialisierung anhand physischer Eigenschaften, die keine Rücksicht nimmt auf die Persönlichkeit und die Sozialisation der Person und kann damit als Reaktion auf die gleichsam von der französischen Rechten übernommenen differentialistischen Debatten der 1970er und 1980er Jahre in Frankreich gewertet werden. ${ }^{22}$ Fanny möchte in das Zentrum vordringen, aus dem sie bisher ausgeschlossen war, weil sie es als ihren natürlichen Ort empfindet. Dieses Zentrum ist dabei jedoch nicht Paris oder eine andere große französische Stadt, es liegt vielmehr in der „France profonde“, in der dörflich geprägten Provinz.

Das Herz eines Landes wird meist nicht in der Hauptstadt angesiedelt, sondern auf dem Dorf. Im Falle Frankreichs lässt sich die Bedeutung der ländlichen Regionen auch insofern gut ermessen, als das bekannteste Chanson der deutschen Okkupations- und Nachkriegszeit, das sich bis heute großer Popularität erfreut, gerade das dörfliche Idyll beschwört. So lautet der Refrain von Charles Trenets „Douce France“:

\section{„Douce France}

Cher pays de mon enfance

Bercée de tendre insouciance

Je t'ai gardée dans mon cœur!

Mon village au clocher aux maisons sages

Où les enfants de mon âge

21 Die Vertraulichkeit und den Vergleich mit anderen, die nicht der von ihr ersehnten Familie angehören, empfindet Fanny als unangenehm. So empfindet sie es als zudringlich, dass sie auf der Busfahrt aus dem Dorf ihres Vaters von einigen Frauen im Bus, deren Sprache sie einst zu kennen meinte, erkannt wird. Für Fanny sind diese Frauen, die sie doch zu kennen behaupten, „les figures d'un monde ennemi, aimables, voulant l'attirer, la faire leur“ (S. 44). Fanny hingegen hat keinerlei Erinnerung an die freundlichen Frauen. Ihre Erinnerung, so erweist sich auch an dieser Stelle, ist keineswegs so gut wie sie behauptet, sondern speichert vielmehr nur diejenigen Informationen, die sie aus ihrem ursprünglichen Kontext herausheben.

22 Siehe hierzu Brubaker 2007: 172ff. 
Ont partagé mon bonheur

Oui je t'aime

Et je te donne ce poème

Oui je t'aime

Dans la joie ou la douleur.“23

Das Glück, an das man sich auch in Krisenzeiten erinnern soll, liegt folglich in dem geradezu prototypischen Dorf. Die Evokation dieser Idylle der Kindheit sollte der Bevölkerung in Zeiten des Krieges Mut machen. Es ist sicherlich nicht als Zufall zu werten, dass die Dörfer, durch die NDiaye ihre Protagonistin schickt, sich nicht nur untereinander ähneln; vielmehr weisen sie auch mit dem von Trenet skizzierten Dorf große Ähnlichkeit auf, insbesondere durch die Tatsache, dass Fanny dort in der Tat ihre in ihren eigenen Erinnerungen glückliche Kindheit mit den anderen Kindern ihres Alters ihren Cousins und Cousinen - geteilt hat. Aus diesem Paradies ihrer Kindheit, das sie, wie es das Lied verlangt, ,dans la joie ou la douleur“ auch angesichts der größten Grausamkeiten ihrer Verwandten liebt, fühlt sie sich vertrieben. Hier, im Herzen Frankreichs und nicht an dessen Peripherie will sie aufgenommen werden und sich zugehörig fühlen dürfen.

Diese Zugehörigkeit, die der Text deutlich als das Recht auf Einbürgerung markiert, ist aber nichts, das als rein administrativer Akt zugestanden wird. Die Vergabe behält sich die dörfliche Bevölkerung vor. Wie die Kriterien für die Aufnahme dabei lauten, bleibt für Fanny opak. ${ }^{24}$ In einer zent-

23 Charles Trenet: „Douce France“ (1941). Dieses Lied, mit dem Trenet „die Einheit von Territorium (pays), Gesellschaft in Form von dörflicher Gemeinschaft (village), Schule (école) und Kirche (clocher) als Grundlage seiner französischen Identität“ beschwört (Adelheid Schumann 2000: 180), wurde 1985/86 von Carte de séjour, einer aus Kindern der Immigration bestehenden Band um den Sänger Rachid Taha, erneut aufgenommen, diesmal in einer Mischung aus arabisierenden Rhythmen und französischem Pop. Damit, so auch Schumann, reklamiere die zweite Migrantengeneration das kulturelle Erbe Frankreichs für sich, was in Frankreich nicht nur auf positive Reaktionen stieß.

24 Vgl. hierzu die Untersuchungen von Fanny Colonna zur Beurteilung von angehenden Lehrern im kolonialen Algerien (1893-1939): „On the path of acculturation, there was a boundary that must not be crossed [...]. But if violations of this 
ralen Szene des Textes sucht sie nach ihrer, Verwandlung' im Rathaus des Dorfes um ihre Einbürgerung nach. Der frisch gewählte Bürgermeister sieht darin kein Problem, wird allerdings von seiner Sekretärin, die bereits wesentlich länger im Amt ist, sofort zur Ordnung gerufen:

„,Oh, c'est très compliqué, murmura la secrétaire [...]. Il n'est pas donné à tout le monde de pouvoir le [d. h. Bürgerin des Dorfes] devenir, certes non. Ici, nous appliquons les lois anciennes. ‘

J'ignorais cela, s'étonna le maire.

,Nous n'appliquons les lois nouvelles, plus simples, que lorsque nous sommes sûrs de la fiabilité de la personne à qui nous avons affaire, expliqua doctement la secrétaire. Or, tant de rumeurs diverses circulent au sujet de cette demoiselle, contradictoires, indébrouillables, que je crois bon, si vous ne vous y opposez pas, de soumettre sa demande à la rigueur des vieilles lois. ““ (S. 226)

Während die Cousins, so ein späterer Vorwurf der Familie, sich an ,quelques règles simples“ (S. 289) hielten und ihr damit Ehre machten, gelten für Fanny andere Bestimmungen. Die Cousins, so wird deutlich, fühlen sich ebenso selbstverständlich als Teil der Familie wie ihnen auch die Regeln, nach denen sie ihr dienen, implizit geläufig sind. Für Fanny hingegen gelten keine „,règles“, sondern vielmehr „les vieilles lois““25, die damit scheinbar eine andere Stufe der Kodifizierung erreicht haben, auch wenn es ihr nicht gelingen will, ihrer in schriftlicher Form habhaft zu werden. Sie scheinen sich Fanny, bei all ihren Bemühungen um ihre Dechiffrierung und Befolgung, immer wieder zu entziehen. Im Gespräch mit dem Geist ihrer Großmutter, die mit einem ironischen Augenzwinkern sozusagen zum „Esprit des Lois“626 wird, beweist diese ihr ihre Unwissenheit:

rule were severely sanctioned, the rule itself was never clearly stated." Colonna 1997: 364.

25 An dieser Stelle werden die Bezüge, die der Text zu den Schriften Kafkas aufweist, offenbar. Zur Untersuchung dieser und anderer intertextueller Bezüge bereitet die Verfasserin eine Publikation vor.

26 Ich spiele hier auf den Titel von Montesquieus geschichtsphilosophischen Hauptwerk De l'Esprit des Lois (1748) an. In NDiayes späterem Roman Mon coeur à l'étroit lebt die Protagonistin, die ebenfalls mit Eingliederungsproble- 
„,Ma petite-fille boit à la fontaine du village, mais en a-t-elle acquis le droit? Lui a-ton jamais dit qu'elle pouvait profiter des services du village comme une véritable habitante?

,Les étrangers eux-mêmes, pensa Fanny, contrariée, les gens de passage sont autorisés à étancher leur soif aux fontaines!

,Certes, mais tu n'es pas une étrangère en visite, expliqua gaiement l'esprit. Ni une habitante ni une visiteuse: que prévoit la loi pour ton cas?‘

,Je l'ignore.

,Vois, tu ignores la loi!““ (S. 291)

Nicht nur das einzelne Gesetz, sondern sogar „la loi“ im Allgemeinen kennt Fanny nicht, trotz ihres unermüdlichen Studiums der ,Familie‘.

Zugleich liegt in den Aussagen des Geistes wiederum eine neue, perfide Regel, die alle bisherigen Mühen obsolet sein lässt: Fanny ist nicht nur keine Bewohnerin des Dorfes, sondern auch kein Gast, der dann ja unter die Regeln der Gastfreundschaft fallen würde. Vielmehr ist sie ein unerwünschtes Drittes, das sich der Bezeichnung entzieht und für das es - jenseits der Notwendigkeit seiner Exklusion - keine Regeln und somit keine Existenzberechtigung gibt.

Fannys Entwirklichung hat damit ein neues Stadium erreicht, das im Roman in konkrete Bildlichkeit umgesetzt wird. Das literarische Mittel hierzu ist der Einbruch des Phantastischen, der bereits vor der Begegnung mit dem Geist der Großmutter begonnen hat: Wie die Großmutter hat auch Fanny diese Schwelle zur Phantastik schon überschritten und ist in einen neuen Zustand übergegangen. Dieser Prozess wird dadurch eingeleitet, dass der Hund ihres Cousins Eugène sie am Tag von dessen Hochzeit im Kreis der Familie zerfleischt. Alle weiblichen Familienmitglieder wirken ungerührt dabei mit, ihre sterblichen Überreste zu beseitigen und auf den Misthaufen zu werfen, um sodann den Apéritif einnehmen zu können.

Fanny ist allerdings nicht dauerhaft tot, sondern wird vielmehr zu einer Wiedergängerin, die ihr gewaltsames Ende als notwendige Selbstaufopferung, als „nécessité d'un tel abandon“ und als ,pénible sacrifice de soimême“ (S. 215) sieht, um endlich in den Kreis der ,Familie“ aufgenommen zu werden.

men zu kämpfen hat, in der „Rue Esprit des Lois“. Zu den Bezügen dieses späteren Romans zu Montesquieus Text siehe auch Ruhe 2012. 
Kurz nach ihrem gewaltsamen Tod findet das statt, was Fanny als ihre Himmelfahrt (,,assomption“, S. 217) bezeichnet. Der Himmel ist für sie die Aufnahme in die ,Familie', mit anderen Worten in das Gemeinwesen der „France profonde“. Tante Colette findet bei einem Waldspaziergang eine nackte Fanny, die sich in signifikanter Weise verändert hat. Sie hat, so lässt sich folgern, ihre identitätsstiftenden Merkmale, die zugleich Grund für ihre Ausgrenzung waren, abgelegt. Sie ist nun „modifié“, „, fort différente de ce qu'elle avait été“, insofern als ,plus rien ne la distinguât, ainsi que nous avions souhaité qu'elle fût autrefois, sans succès tant était mauvais le fond de sa nature." (S. 211) Erst in diesem Moment scheinen Fannys Bemühungen, den Erwartungen ihrer Familie zu entsprechen, endgültig erfolgreich, sie hat in der Tat ihre ,insoutenable singularité“ (S. 300) verloren. Tante Colette erscheint ihre Nichte, die sie gerade noch aus der Familie ausgeschlossen hatte, in diesem neuen Zustand als ,parfaite“ (S. 212). Es zeigt sich allerdings bald, dass sich aus diesem Zustand eine doppelte Ausschließung ergibt. Von der Gemeinschaft an der Peripherie der Großstadt wird sie nun als nicht mehr zugehörig betrachtet. Für die Aufnahme in den ,Himmel“ der „France profonde“, dessen Zugang Tante Colette zu unterstehen scheint, die als Repräsentantin des Gemeinwesens und seiner Werte entworfen ist, reicht Fannys Perfektion jedoch immer noch nicht aus.

Als sie endgültig offiziell um ihre Einbürgerung im Dorf nachsucht, wird sie zurückgewiesen. Kommentierend dazu eröffnet ihr der Geist der Großmutter:

„Eussé-je émis un doute sur ton droit à exister seulement devant cet habitant du village que, très certainement, sans peut-être même t'en apercevoir, tu serais devenue aussitôt invisible à ses yeux [...].“ (S. 292)

Der als Anmaßung gesehene Versuch Fannys, die Zugehörigkeit zum Dorf und zur Familie auf Kosten extremer Selbstaufopferung zu erlangen, scheitert erneut. Fanny hat dabei allerdings in so erheblicher Weise an Substanz verloren und soviel von ihrer eigenen Identität preisgegeben, dass sie nahezu unsichtbar geworden ist. Letztlich wird sie sich völlig auflösen. 


\section{AssimiLATION UND UNSICHTBARKEIT}

Der Zusammenhang zwischen Gesetz und Sichtbarkeit, der hier auf literarischer Ebene umspielt wird, ist noch in anderer Hinsicht interessant: Pap Ndiayes zentrales Argument in seiner Untersuchung zur schwarzen Bevölkerung in Frankreich ist, dass die Antidiskriminierungsgesetzte in paradoxer Weise nicht dazu geführt haben, dass es weniger Diskriminierung gibt; die Tabuisierung habe zwar eine Veränderung der Wahrnehmung bewirkt, aber daraus hätten sich keinerlei positive Rückwirkungen auf die Lebensrealität ergeben. Im Sinne des republikanischen Grundsatzes der égalité ermögliche die Egalisierung auf verbaler Ebene, ethnische Unterschiede lediglich diskursiv verschwinden zu lassen.

Die Gesellschaft, in der Fanny lebt, ist ein literarisch verdichtetes Abbild einer Gesellschaft, die sich gegen Assimilation abschottet. Zuordnungen werden aufgrund von äußerlichen Merkmalen getroffen, die auch Kategorien wie ,Heimat' determinieren. Die Abschottung gegenüber dem Fremden oder Menschen, die als fremd empfunden werden, ihre Exklusion, ist ebenso massiv wie die Forderung nach Assimilation. Selbst die Erfüllung dieser Forderung wird allerdings nicht mit Wohlgefallen aufgenommen, sondern als Versuch der Nachahmung nationaler Eigenheiten gewertet, die nie den Charakter der Echtheit gewinnen kann. Die Assimilation in der hier geschilderten, extremen Form führt zur Vernichtung der Persönlichkeit.

In einer ironischen Schlussvolte kehrt Marie NDiaye die Ausgangssituation des Textes um: So wie Fanny am Tor des Familienanwesens um Einlass bat und unfreundlich empfangen wurde, stehen jetzt ihre Tante Colette und deren Sohn Eugène vor dem prächtigen Haus des Vaters von Fanny und werden dort sehr höflich und etwas überrascht hereingebeten. Das Staunen des Gastgebers steigert sich, als Tante Colette ihn bittet, sich ihres Sohnes anzunehmen, der es bisher im Leben zu nichts Rechtem gebracht habe. In der zwischenzeitlich auch in der „France profonde“ eingebrochenen Moderne erweist sich das bisherige Wertesystem als überholt. Orientierung für die im Sohn personifizierte Zukunft des Landes muss jetzt bei den bisher verachteten, an die Peripherie verdrängten Anderen gesucht werden. 


\section{LITERATUR}

Brubaker, Roger. Ethnizität ohne Gruppen. Hamburg: Hamburger Edition 2007.

Colonna, Fanny: „Educating Conformity in French Colonial Algeria“. In: Cooper/Stoler 1997: S. 346-370.

Cooper, Frederick /Stoler, Ann Laura (Hg.): Tensions of Empire. Colonial Cultures in a Bourgeois World. Berkeley u.a.: University of California Press 1997.

Lagrange, Hugues. Le déni de la culture. Paris: Seuil 2010.

Ndiaye, Pap. La condition noire. Essai sur une minorité française. Paris: Calmann-Lévy 2008.

Ruhe, Cornelia. „Le corps de la ville. L'espace urbain chez Marie NDiaye“. In: Dünne, Jörg/ Nitsch, Wolfram (Hg.): Scénarios de l'espace. Littérature, film et techniques de déplacement dans l'espace urbain. ClermontFerrand: Presses universitaires Blaise Pascal 2012.

Schumann, Adelheid. ,,Douce France“: die Aneignung der mémoire collective Frankreichs durch die Immigranten der zweiten Generation“. In: Frankreich Jahrbuch 2000. S. 179-186.

Stoler, Laura Ann. „Sexual Affronts and Racial Frontiers: European Identities and the Cultural Politics of Exclusion in Colonial Southeast Asia“. In: Cooper/Stoler 1997. S. 198-237. 
Bereitgestellt von | Universitätsbibliothek Mannheim

Angemeldet

Heruntergeladen am | 26.10.17 09:52 